\title{
Are time delays always destabilizing? Revisiting the role of time delays and the Allee effect
}

\author{
Masha Jankovic ${ }^{1} \&$ Sergei Petrovskii \\ Department of Mathematics, University of Leicester, \\ Leicester, LE1 7RH, U.K.
}

\begin{abstract}
One of the main challenges in ecology is determining the cause of population fluctuations. Both theoretical and empirical studies suggest that delayed density dependence instigates cyclic behavior in many populations, however underlying mechanisms through which this occurs are often difficult to determine and may vary within species. In this paper we consider single species population dynamics affected by the Allee effect coupled with discrete time delay. We use two different mathematical formulations of the Allee effect and analyze (both analytically and numerically) the role of time delay in different feedback mechanisms such as competition and cooperation. The bifurcation value of the delay (that results in the Hopf bifurcation) as a function of the strength of the Allee effect is obtained analytically. Interestingly, depending on the chosen delayed mechanism, even a large time delay may not necessarily lead to instability. We also show that, in case the time delay affects positive feedback (such as cooperation), the population dynamics can lead to self-organized formation of intermediate quasi-stationary states. Finally, we discuss ecological implications of our findings.
\end{abstract}

Keywords delayed density dependence; competition; cooperation; population oscillations; intermediate states

\footnotetext{
${ }^{1}$ Corresponding author. E-mail: mj116@le.ac.uk
} 


\section{Introduction}

The classical theory of population dynamics predicts that the smaller the population (or the lower its density), the less individuals will suffer from constraints of intraspecific competition. The fewer individuals there are, the more resources would be available, and steadily the population would increase in size. Though this enhanced resource availability would ostensibly benefit the population, such a population would lack conspecifics (advantages of which include predator saturation or dilution, cooperative predation or resource defense and social thermoregulation, e.g. see Stephens et al. 1999) which may lead to a decrease in the population's fitness. This phenomenon, termed the Allee effect, was first described in the 1930 's, and has become a topic of much interest over the last 25 years, largely in light of the concerns over conservation and the problems of already endangered or rare species (Dennis 1989, Courchamp et al. 2008). The Allee effect refers to a positive relationship between (any component of) individual fitness and population density (Stephens et al. 1999). The per capita growth rate is nonmonotonic and can even become negative at particularly low values of population density (termed as the strong Allee effect), resulting in a critical density known as the Allee threshold below which population extinction is likely.

Mechanisms through which the Allee effect appears are abundant, and to name just a few, include inbreeding depression, absence of cooperative feeding, failure to satiate natural enemies, failure in mate finding and temporal asynchrony in reproductive maturing between sexes (known as protandry); see Courchamp et al. (1999), Stephens et al. (1999). One way in which individuals gain protection from predators is through cooperative strategies such as aggregation. By grouping, the population reduces predation risk through dilution effects; the larger the group size the less chance that an individual may be harmed. At the same time, aggregation can be costly and overshadowed by deleterious consequences such as a paradoxical increase in exposure to predators, as larger groups are easily detected. Furthermore, it can lead to overcrowding and an increase in intraspecific competition (Griffiths et al. 2003). These negative effects can be counteracted through behaviour such as parental care, which restores the positive aspects and need for animal grouping (Nakaoka et al. 2009). A less appreciated and frequently overlooked mechanism through which Allee effects emerge are predator-prey interactions. It has been shown, that size-selective predation on prey regulated through density-dependence leads to a positive relationship between predator density and population growth, through intraspecific competition in the prey population (De Roos and Persson 2002, De Roos et al. 2003, van Kooten et al. 2005). Such an Allee effect is known as "emergent", as it results from the predator induced changes in prey size distribution and is essentially a consequence of the feedback of predator feeding on its own performance.

Despite Allee effects usually being difficult to detect and quantify in natural populations (but see Vercken et al. 2011), the variety of mechanisms and affected ecological processes suggest their prevalence. Moreover, the current state of research indicates ample empirical evidence (albeit often indirect) of the ubiquity of Allee effects in a diverse range of taxa and ecosystems (Courchamp et al. 2008, Courchamp et al. 1999). Along with stochasticity, the Allee effect is particularly influential in biological invasions (Fagan et al. 2002, Petrovskii et al. 2002, 2005a), as it may cause longer lag times (Petrovskii et al. 2005b), slower spread (Lewis and Kareiva 1993) and decrease the probability of establishment which may lead to underestimation of invasion risk posed (Taylor and Hastings 2005). Further consequences include impacts on 
distribution of invasive species (Morozov et al. 2006), metapopulation fluctuations, predatorprey interactions, parasite transmission (Kramer et al. 2009), exploitation management and biodiversity preservation (Taylor and Hastings 2005, Courchamp et al. 1999). It is thought that the collapse of cod (Gadus morhua) in the Northwest Atlantic might be an unfortunate consequence of emergent Allee effects, as these populations are not known to exhibit mechanisms commonly associated with positive density dependence (De Roos and Persson 2002). Allee effects also form a crucial aspect of biological control (e.g. through release of sterile insects, cf. Lewis and van den Driessche 1993) and are increasingly exploited as the "Achilles heel" of biological invasions (Liebhold and Tobin 2010, Dennis 1989).

The ways to include the Allee effect into mathematical models of population dynamics are well understood. The resulting models are often described by differential equations (Murray 1989, Kot 2001) hence assuming that the processes shaping the dynamics are taking place at the same time. However, processes in ecology (as well as in nature in general) are rarely instantaneous (Hutchinson 1948, Maynard Smith 1974). As it was pointed out by Kuang (1993), "time delays occur so often (in almost every situation) that to ignore them is to ignore reality". Aiming to create a somewhat more realistic description, one should include a time delay into the model, which results in a delay differential equation. Delay differential equations have been of sufficient importance in modeling real life phenomena, and arise in various practical applications such as population dynamics (most often accounting for maturation/gestation periods), immunology (incubation/latent periods) and physiological and pharmaceutical kinetics (Cheyne-Stokes respiration, glucose-insulin regulation, blood pressure oscillations), etc. Time delays in feedback mechanisms tend to destabilize positive equilibria and lead to oscillatory behavior. Thus, delay differential equations are capable of generating more complex and rich dynamics compared to ordinary differential equations (Ruan 1995, Cooke and Grossman 1982). Indeed, population densities of many species are known to fluctuate nearly periodically over time (cf. Turchin 2003), a phenomenon to which delayed density dependence may provide an explanation.

Consequently, the conditions under which time delay can lead to an instability are studied in much detail. Interestingly, there is some evidence that the Allee effect can have an effect on the system's dynamics opposite to that of the time-delays, i.e. increasing stability of the positive equilibrium rather than decreasing it. In particular, Gopalsamy and Ladas (1990) proposed a single species model in which population growth is damped by the Allee effect and per capita growth is subject to time delays. Under certain conditions that included the case of a weak Allee effect (but not the strong Allee effect), they showed the positive equilibrium was globally attractive. Subsequent studies by Cao and Gard (1995) and Liz et al. (2003) provided conditions of global asymptotic stability; however, if a delay exceeds a critical value, the solutions oscillate around the positive equilibrium. Freedman and Gopalsamy (1986) obtain conditions for local stability for a class of models including the delayed weak Allee effect. Recently, it was shown that a mere inclusion of a (non-delayed) Allee effect can have a stabilizing effect on the positive equilibrium compared to Hutchinson's model (Merdan et al. 2009), in the sense that the steady state is stable under less restrictive conditions. Moreover, the conditions become less and less restrictive - hence the stabilizing effect becomes more prominent - with increasing strength of the Allee effect (Merdan et al. 2009).

Regarding the effect of various factors on the system's stability, we also mention here the work 
by Beddington and May (1975) who showed that the effect of the time delay can be arguable and delicate. We however point out here, that their context of stability differs principally from other studies, including ours. Namely, they showed that, although an increase in the value of time delay causes the solution to start oscillating around the upper equilibrium, simultaneously it spends more time in the vicinity of the lower, trivial, otherwise unstable equilibrium. Thus, time delays can slow the growth of instabilities and can therefore be interpreted as having a "stabilizing" effect on unstable stationary points (Beddington and May 1975).

In spite of several papers having been published about the interplay between the time delay and the Allee effect, there are a few questions that have never been addressed. Note that most of the previous research focuses on the weak Allee effect. On the contrary, here we consider both cases of weak and strong Allee effects. Merdan et al. (2009) obtain results for a general class of Allee functions, however our chosen parametrizations are different and do not satisfy their imposed restrictions. That raises an issue of the structural sensitivity (Fussmann and Blasius 2005) of models with regard to parametrization of the time-delayed Allee effect. In this paper, we show that the interplay between the Allee effect and time delay can be non-trivial and counterintuitive depending on the choice of delayed process(es). The remainder of the paper is organized as follows. In Section 2 we introduce our modeling frameworks and consider the effects of different time delay terms. Results presented include both numerical simulations and analytical results. Then, in Section 3 we address possible ecological applications of our models and outline what ecological implications our findings may leave.

\section{Models and results}

Mathematical modeling is a powerful research tool in theoretical ecology. The approach to model building can be very different though. Depending on the purposes of the study, there have been two different streams (Maynard Smith 1974). In case the purpose of modeling is to predict the ecosystems state, the model is expected to explicitly include many details of the ecosystem structure. This approach is called predictive modeling. Models arising in this way can be very complicated. Alternatively, the purpose of the study can be to understand some basic features of the ecosystem, e.g. to identify the factors potentially responsible for a population outbreak or population fluctuations, but not necessarily to predict their development quantitatively. In this case, the corresponding models can be pretty simple. This approach is conventionally called conceptual modeling. It is this approach that we use in this paper as our aim here is to reveal some general tendencies rather than to provide any kind of detailed and/or predictive description. It has been pointed out (Levins 1968) that, when studying systems as complex as ecosystems, one should not look for inferences that are likely to be true for all cases. Instead, one should rather look for the causes of differences between different species or different systems. To address this sort of question, we need a model which is as simple as possible (Maynard Smith 1974).

Correspondingly, under the assumption of spatial homogeneity, we describe the dynamics of a single species population by the following equation:

$$
\frac{d U(t)}{d t}=G(U(t))=U(t) F(U(t)),
$$

where $U(t)$ is the population density at time $t, G(U)$ the rate of change of population density 
due to reproduction and mortality and $F(U)$ is the per capita growth rate. If population growth is damped by the Allee effect, the per capita growth rate is dome-shaped and can be described by a square polynomial (Lewis and Kareiva 1993, Amarasekare (1998a), Amarasekare (1998b), Courchamp et al. (2008)):

$$
F(U)=\gamma(K-U)(U-\beta),
$$

where $K$ the carrying capacity and $\gamma$ is the coefficient proportional to the maximum per capita growth rate (cf. Lewis and Kareiva 1993) which we call, for convenience, the characteristic growth rate. Parameter $\beta$ is the so-called Allee threshold or threshold density and can be regarded as a measure of the strength of the Allee effect, so that the Allee effect is called strong if $\beta>0$ and weak if $-1<\beta<0$.

Accordingly, our baseline non-delayed model is as follows:

$$
\frac{d U}{d t}=\gamma U(K-U)(U-\beta)
$$

Equation (3), albeit very simple, accounts for the two dominant feedback mechanisms: positive, arising from cooperation at low population densities, and negative occurring at high densities due to competition; see Fig. 1. Therefore, in the chosen parametrization (2) we can loosely associate cooperation and competition with factors $(U-\beta)$ and $(K-U)$, respectively.

Once the delay $\tau$ is taken into account, the generic Eq. (1) turns into

$$
\frac{d U(t)}{d t}=G(U(t), U(t-\tau))=U(t) F(U(t), U(t-\tau)),
$$

where, similarly to the above, $G$ is a cubic polynomial but the delay can enter different terms depending on the type of the feedback.

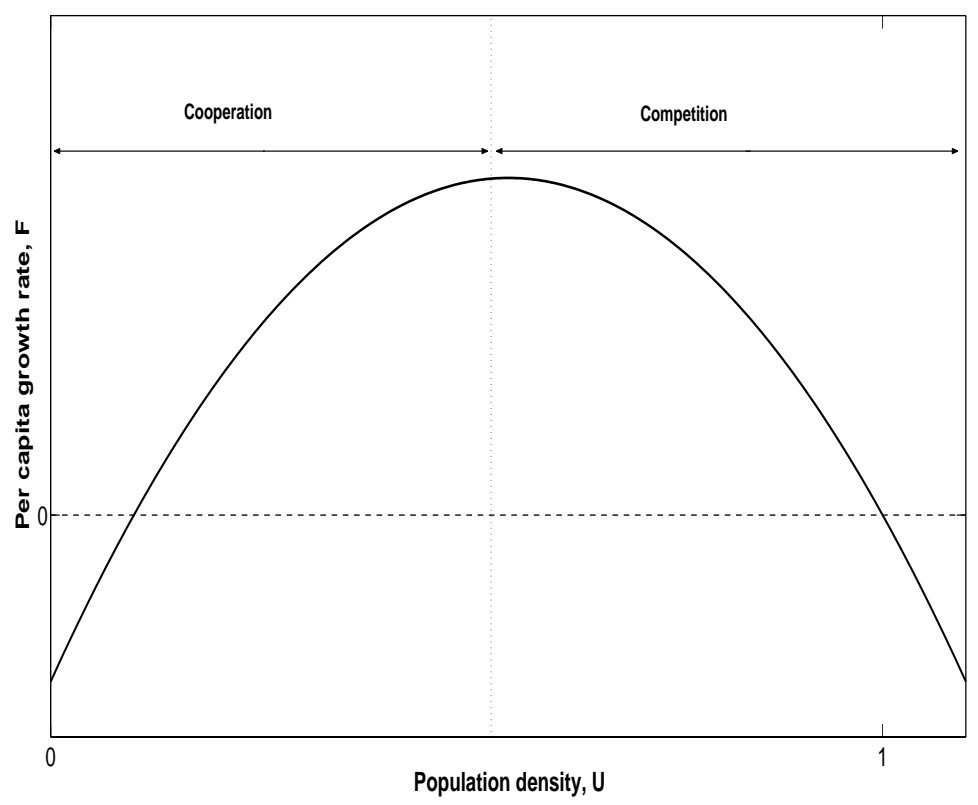

Figure 1: A sketch of the per capita growth rate $F(U)$ in the case of the strong Allee effect. 
In population ecology, typical biological regulating mechanisms are competition for food and territories, amongst others (Berryman et al. 1987), thus we begin by including time delay into the competition term, obtaining a model comparable to Hutchinson's equation (Hutchinson 1948):

$$
\frac{d U}{d t}=\gamma U(t)(U(t)-\beta)(K-U(t-\tau))
$$

where $\tau$ is the time delay.

Throughout the paper we have scaled both the carrying capacity $K$ and the characteristic growth rate $\gamma$ to unity, which implies the corresponding scaling for the variables $U$ and $t$. Equation (5), as well as other delay differential equations (see Eqs. (6) and (10-13) below) were solved by finite differences, in particular by the explicit Euler scheme. The time step was chosen to be sufficiently small, ensuring the accuracy and reliability of results, $\Delta t=0.01$. Furthermore, we have checked the reliability of our solutions with Matlab's in-built solver for discrete delay differential equations (dde23). Contrary to ordinary differential equations, in which defining initial conditions was sufficient, delay differential equations require us to define the population's "history", i.e. $U(t)$ for $-\tau<t<0$. In all simulations, the history was chosen to be a constant function $U=U_{0}=0.5$ (except for $\beta=0.5$ where we choose $U_{0}=0.6$ to ensure population survival). The Allee threshold density $\beta$ is regarded as a controlling parameter and its value is varied between -1 and 0.5 , which obviously include both cases of the Allee effect, i.e. weak for $-1<\beta \leq 0$ and strong for $0<\beta<0.5$. We choose the time delay $\tau$ as the second controlling parameter and observe the system's response to its variation. Following extensive computer simulations, we then summarize our findings as a map in the parameter plane $(\tau, \beta)$, Fig. 2 .

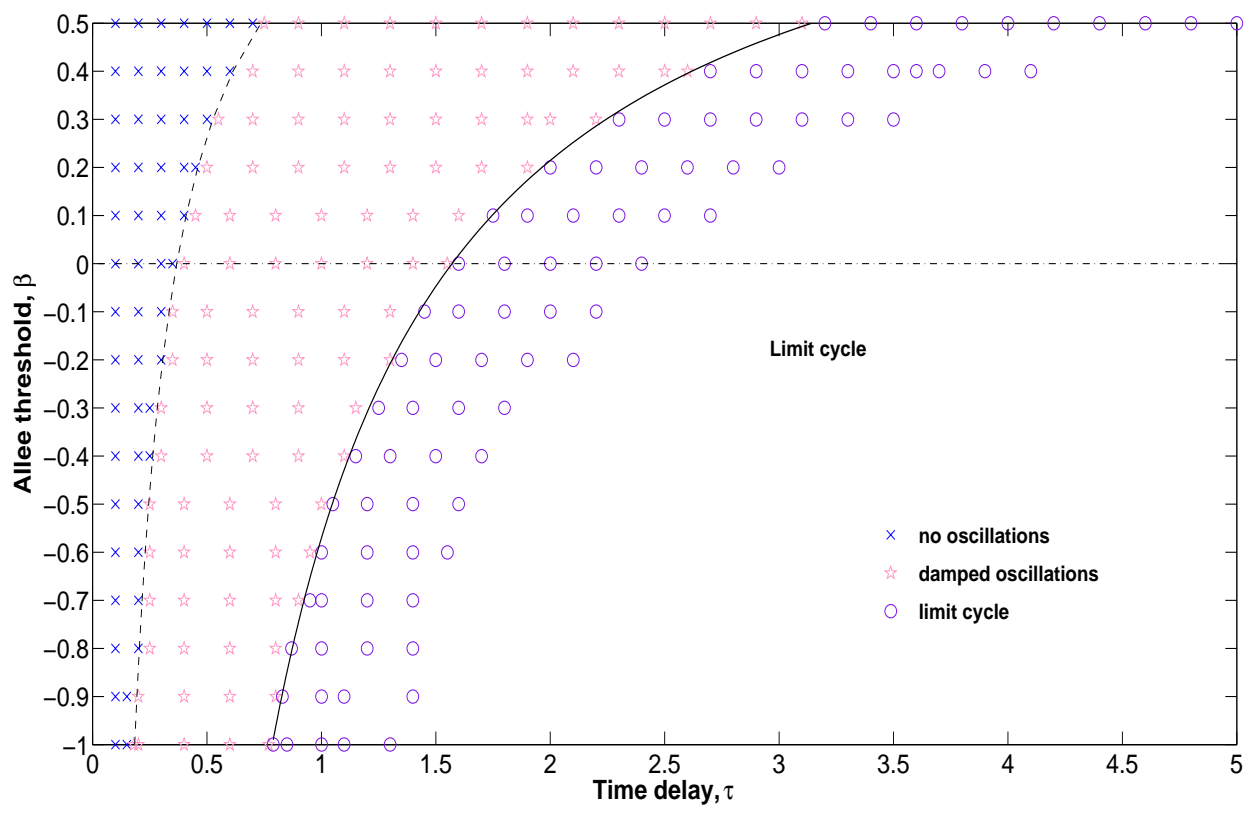

Figure 2: Map of different scenarios as explained by the figure legend for model (5). The solid line corresponds to the analytically calculated Hopf bifurcation curve (see Appendix A), the dotted line corresponds to the analytically calculated loss of monotonicity curve (see Appendix B), while the dash-dotted line acts as a visual aid to separate the weak and strong Allee effect. 
Figure 2 shows the succession of different dynamical responses for a population subject to delayed competition, as descried by Eq. (5). Some typical system's dynamics is shown in Fig. 3. For a given Allee threshold and a small time delay, the system quickly approaches the equilibrium state $U=1$ with $U(t)$ being a monotonously increasing function (not shown). An increase in $\tau$ causes the solution to lose monotonicity (when $\tau$ exceeds the critical value shown by the dashed curve in Fig. 2) through the appearance of damped oscillations (Fig. 3, left). By further increasing $\tau$, the system passes through the Hopf bifurcation (shown by the solid curve in Fig. 2) leading to the occurrence of limit cycles (Fig. 3, right). As the Allee threshold $\beta$ increases, the population appears to be more stable as larger values of the time delay are needed to destabilize it. Also, as we have observed in our simulations, the population always persists and no extinction occurs even for a large time delay. Note that both the solid and dotted curves in Fig. 2 are calculated analytically (see Appendices A and B) and are in excellent agreement with our numerical results.
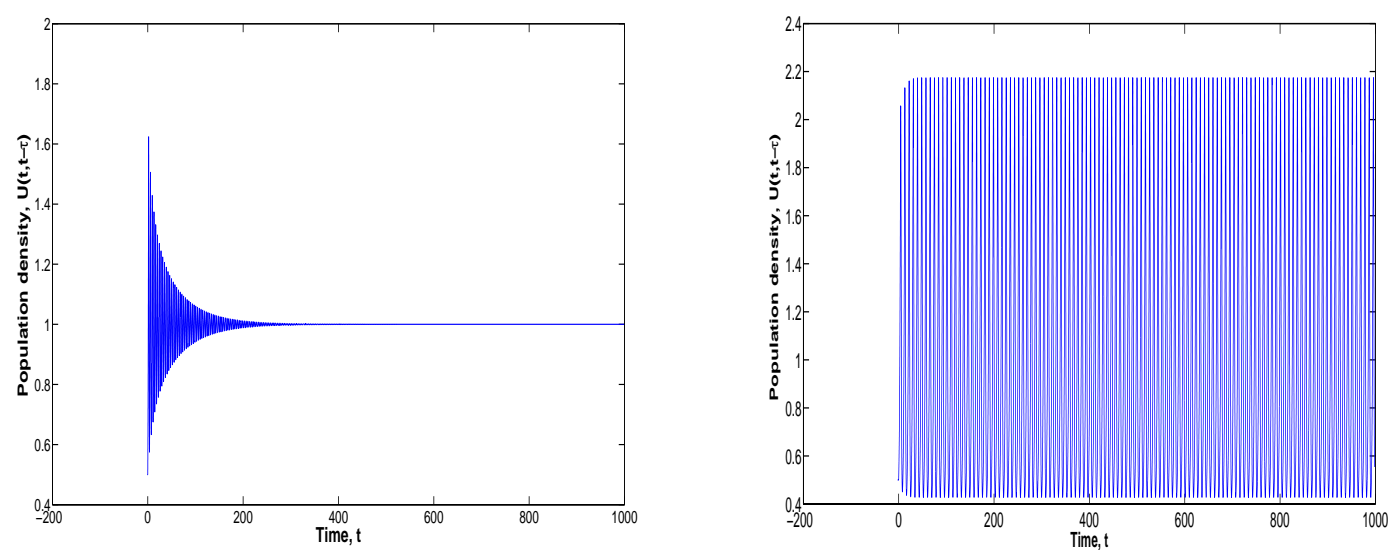

Figure 3: The population density vs time as predicted by model (5). Parameters are $\beta=$ $0.7, \tau=0.9$ (left) and $\beta=0.1, \tau=2$ (right).

As a next step we consider the effect of time delay on cooperation. Delayed cooperation in a population (when time delay is included into positive density dependence) can be modeled as

$$
\frac{d U}{d t}=\gamma U(t)(U(t-\tau)-\beta)(K-U(t))
$$

Contrary to model (5), increasing the time delay had no destabilizing effect on solution behavior, apart from minute changes in the slope (Figure 4). This was intuitively expected, as positive density dependent mechanisms do not regulate population density. For a sufficiently large time delay solutions monotonously approach the equilibrium $U=1$, but at a lower rate.

An apparent disadvantage of the baseline model (3) with the growth rate described by a cubic polynomial is that it accounts for the effects of competition and cooperation in a mixed, multiplicative way. Correspondingly, Petrovskii et al. (2008) have proposed an alternative, additive form of the per capita growth function $F(U)$ :

$$
F(U)=f(U)-m-A(U)
$$




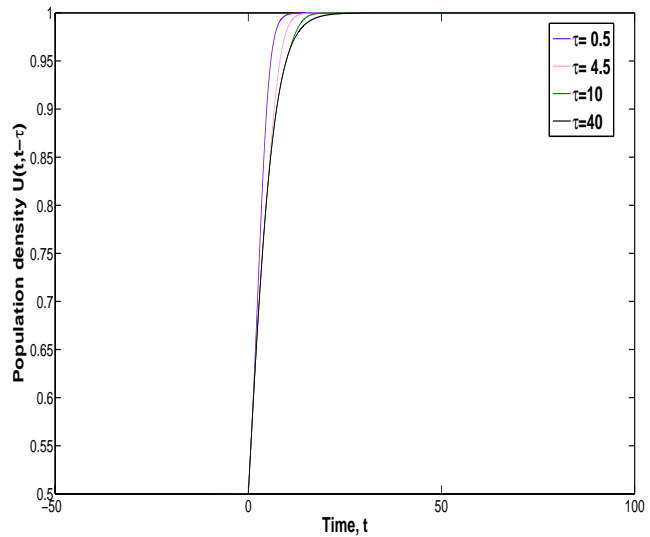

Figure 4: Solutions of model (6). Simulations presented are for the strong Allee effect with $\beta=0.2$. The corresponding time delays are given in the figure legend. Qualitatively similar dynamics are observed for the weak Allee effect (not shown here for the sake of brevity).

where $f(U)$ accounts for population multiplication due to reproduction, $m$ is natural (density independent) mortality and $A(U)$ represents density dependent mortality (e.g. due to cannibalism), which is of significance in high density populations. Unlike the previously introduced multiplicative form of the Allee effect, this additive form avoids the interference of different biological processes. The population dynamics can then be modeled by:

$$
\frac{d U}{d t}=U(-m+f(U)-A(U))
$$

in which the particular parametrization of functions depends on the system modeled.

Obviously, for different choices of functions $f$ and $A$, model (8) can have various properties. Here we consider the growth term as $f(U)=a U$ and the density dependent mortality as $A(U)=\gamma U^{2}$ where $a$ and $\gamma$ are parameters. We then assume that the natural mortality rate $m$ is proportional to the threshold density $\beta, m=\gamma \beta$. Since $\beta$ has the meaning of the survival threshold, this is not completely unrealistic. Finally, in order to make model (8) directly comparable with (3) and to avoid additional parameters, we assume that $a=\gamma(1+\beta)$. Equation (8) then takes the following form:

$$
\frac{d U}{d t}=\gamma U\left(-\beta+(1+\beta) U-U^{2}\right)
$$

(in appropriately chosen dimensionless units). As before, $\gamma$ is the characteristic growth rate, and $\beta$ is the Allee threshold. Without time delay, model (9) is therefore identical to (3). However, different terms now possess different meaning and hence the time delay can be included differently. As the term $(1+\beta) U$ apparently provides a positive feedback on the population growth, we associate it with cooperation. The term $U^{2}$ provides a negative feedback and hence should be associated with competition.

Since time delays in population models often account for maturation or gestation periods, we firstly include the time delay into the growth term:

$$
\frac{d U}{d t}=\gamma U(t)\left(-\beta+(1+\beta) U(t-\tau)-U^{2}(t)\right) .
$$


Following stability analysis (see Appendices A and B), one readily concludes that this inclusion of time delay should not affect population dynamics, as the equilibrium retains its stability. However, numerical simulations reveal interesting population dynamics resulting in the selforganized formation of intermediate quasi-stationary states; see Fig. 5. Namely, after a period of fast growth, the population density approaches an intermediate quasi-equilibrium at the close vicinity of which it can remain for a considerable time. Subsequently, the population density leaves the vicinity and once again shows a fast growth before reaching another quasi-equilibrium. This pattern repeats itself until the system reaches its stable equilibrium $U=1$ in the large-time limit, thus resulting in a step-like structure (Fig. 5, left column). As the time delay $\tau$ increases, the system remains at these "resting states" for longer and the transition between them becomes faster, making the step structure more prominent. Also, by increasing the time delay, more time is needed for the population to reach its equilibrium. Note that the values of $\tau$ used in Fig. 5 are considerably larger than in the models considered above. We mention here that somewhat similar dynamics were observed in So et al. (2001) but for a more complex model involving
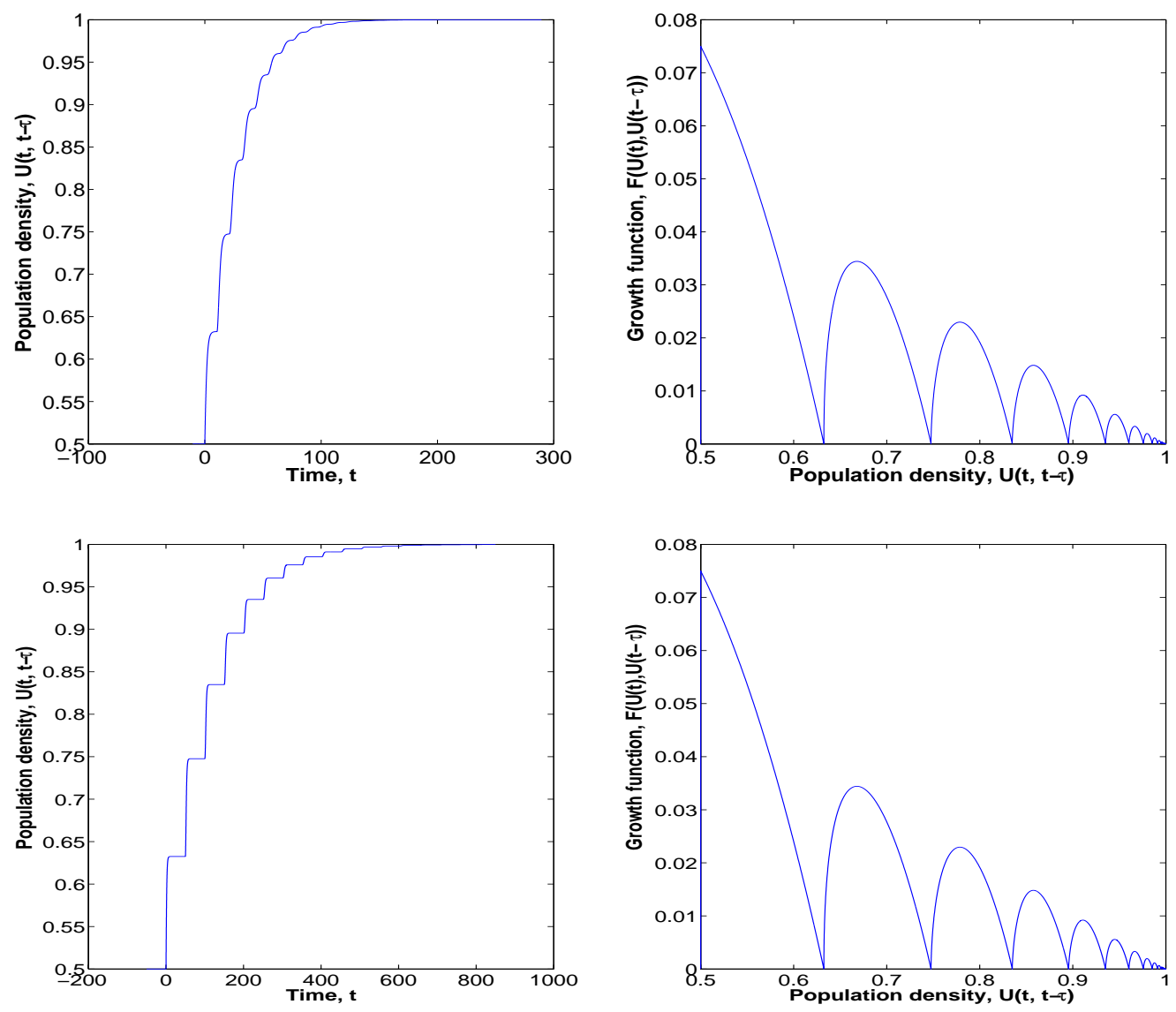

Figure 5: Solution behaviour as predicted by model (10); top for $\tau=10.5$ and bottom for $\tau=50$, the Allee threshold is $\beta=0.2$. The figures on the left show the population density vs time whilst the figures on the right show phase plane structure (growth function $G$ vs population density). By increasing the time delay $\tau$ the step-like structures become more prominent (note the different range used for the $t$-axis in the top and bottom rows). 
a structured population on two patches. However, contrary to our results their solutions lose monotonicity for a critical dispersal rate.

Using the additive model (9), populations subject to delayed competition can be modeled as

$$
\frac{d U}{d t}=\gamma U(t)(-\beta+(1+\beta) U(t)-U(t-\tau) U(t)) .
$$

Figure 6 shows the parameter plane $(\tau, \beta)$ of the model (11) where different symbols corresponds to different regimes of system's dynamics, as was obtained in computer simulations. The Hopf bifurcation curve (solid line) is a decreasing function and matches well with analytical results. For a given Allee threshold, an increase in time delay gradually leads to the loss of monotonicity and to the appearance of damped oscillations. A further increase in $\tau$ causes the system to lose stability and exhibit limit cycle behaviour. An obvious exception occurs at $\beta=-1$ as the system remains stable for any $\tau$ (see Appendix A). A key feature of this model is that a sufficiently large time delay may drive the population to extinction, only if subject to the strong Allee effect, whilst populations affected by the weak Allee effect persist.

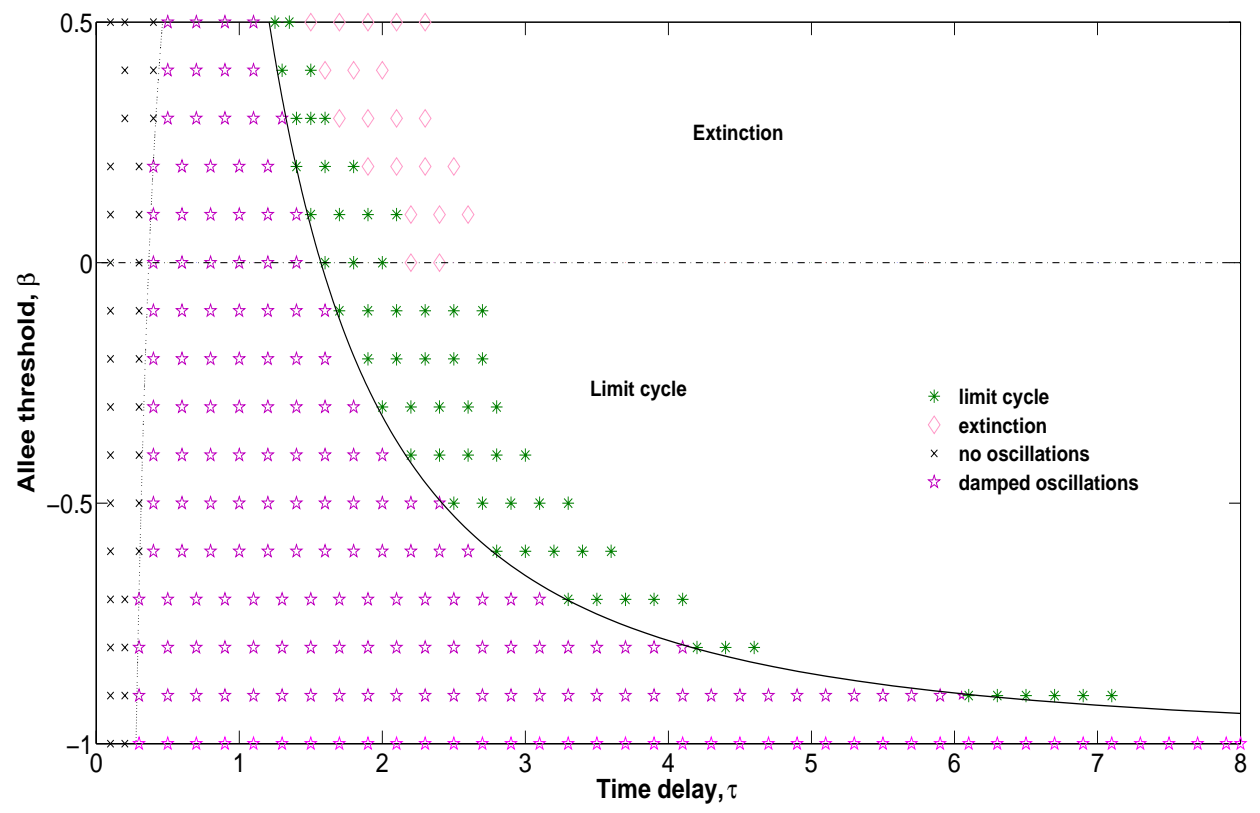

Figure 6: Map of different scenarios as explained by the figure legend for model (11). The solid line corresponds to the analytically calculated Hopf bifurcation curve, the dotted line shows the analytically calculated loss of monotonicity curve, while the dash-dotted line acts as a visual aid to separate the weak and strong Allee effect.

Since Eq. (11) is a heuristic model, it is not immediately clear what the effect of delay on competition exactly is. Therefore, we consider an alternative model where additional mortality may be controlled by a purely delayed mechanism, so that population dynamics is modeled as

$$
\frac{d U}{d t}=\gamma U(t)\left(-\beta+(1+\beta) U(t)-U^{2}(t-\tau)\right) .
$$

The parameter plane of model (12) is structurally similar to that of model (11) whilst the 
bifurcation curve is monotonically decreasing, with a substantially steeper slope (Fig. 7). Again, populations can be driven to extinction only when a strong Allee effect is present.

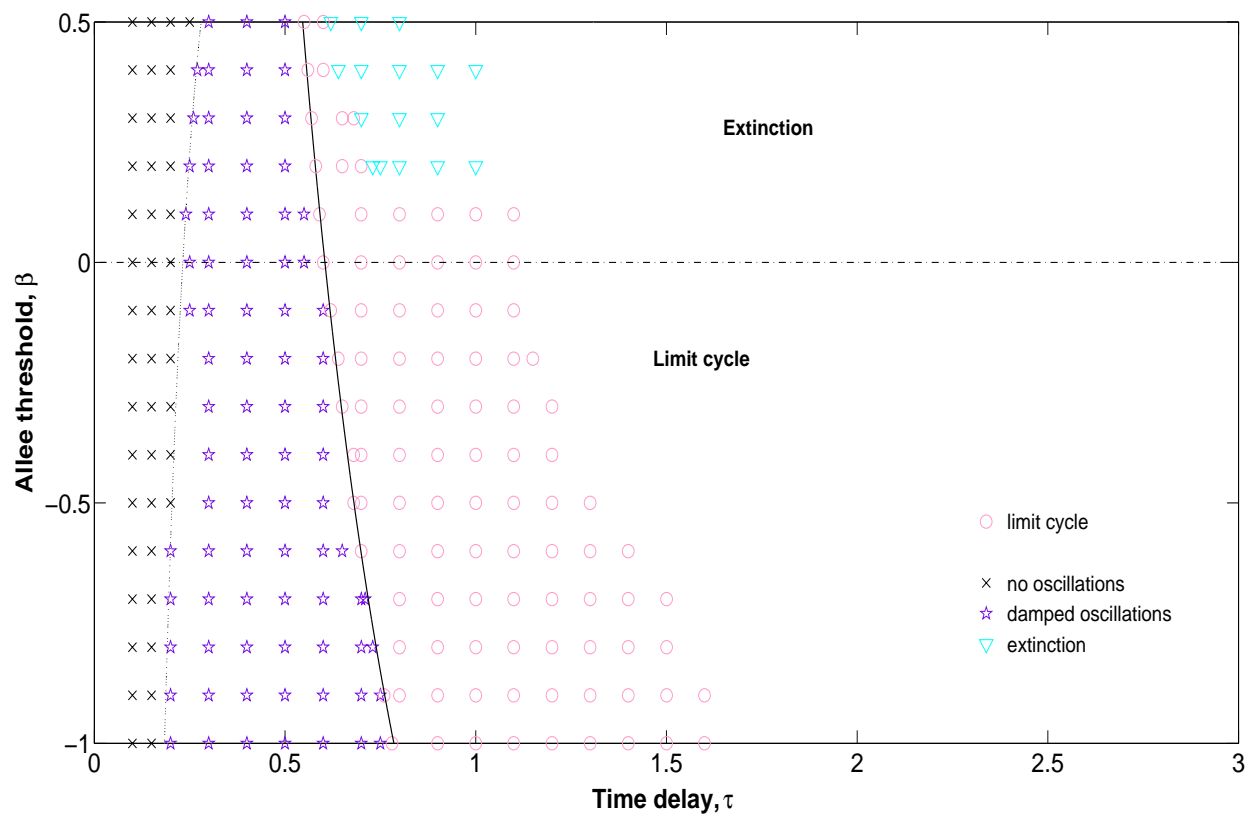

Figure 7: Parameter map of different scenarios as explained by the figure legend for model (12). The solid line corresponds to the analytically calculated Hopf bifurcation curve, the dotted line shows the analytically calculated loss of monotonicity curve, while the dash-dotted line acts as a visual aid to separate the weak and strong Allee effect.

Lastly, we consider the inclusion of both delayed competition and maturation (which is equivalent to delays in both cooperation and competition in the baseline model (3)):

$$
\frac{d U}{d t}=\gamma U(t)\left(-\beta+(1+\beta) U(t-\tau)-U^{2}(t-\tau)\right)
$$

For this model, the map in the parameter space $(\tau, \beta)$ (see Fig. 8) appears to have a structure similar to that obtained in the corresponding multiplicative model (5) (see Fig. 2) where the delay was included only into the cooperation term. This seems to suggest that delay in competition may have a more prominent effect than delayed cooperation. One important difference though is that now, in case of the strong Allee effect $(\beta>0)$ and for sufficiently large time delays (see the top-right corner of the parameter plane), the population can be driven to extinction.

\section{Discussion}

This paper aims to address the interplay between time delays and the Allee effect. Using the single-species context, we study (both analytically and numerically) two different models of population dynamics where the effects of competition and cooperation are taken into account in either a multiplicative or additive way. The conditions for local stability are obtained analytically as a function of the Allee effect strength and are in full agreement with numerical results. We have found that the inclusion of time delay alone does not necessarily confer instability, and 


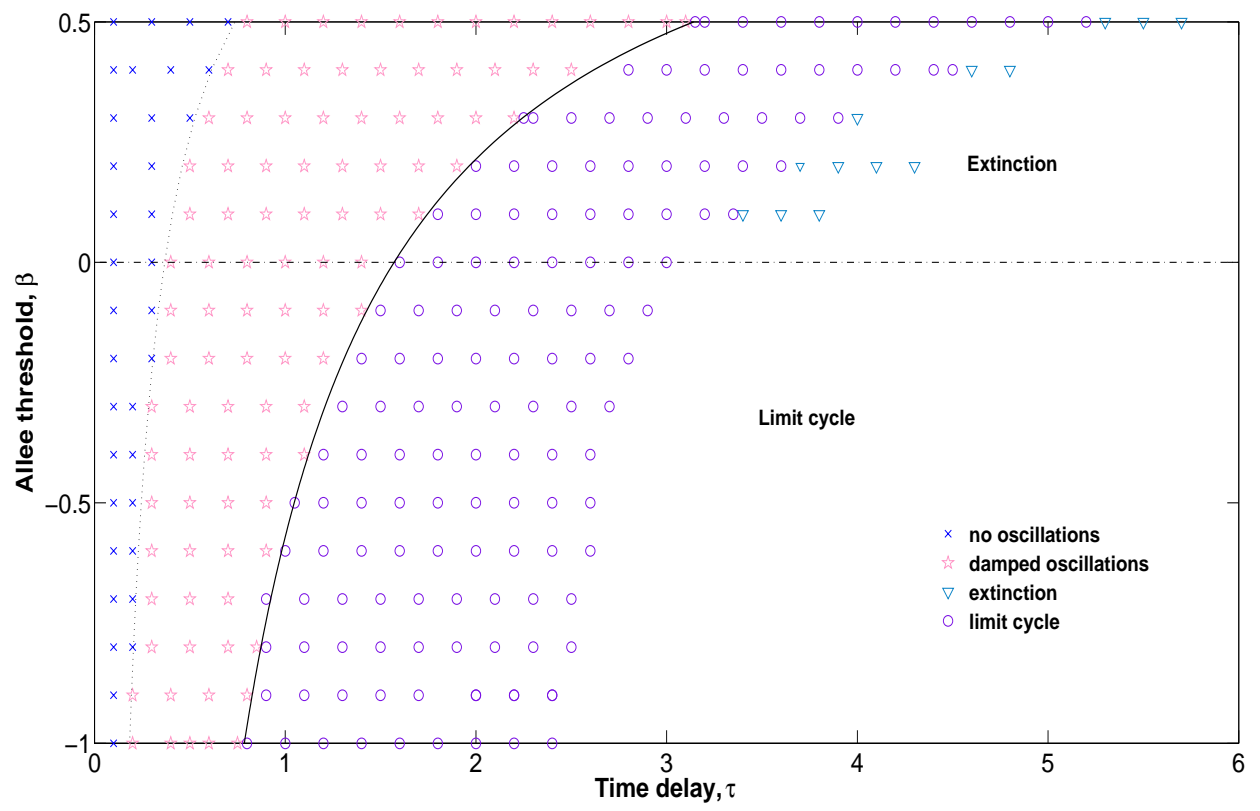

Figure 8: Map of different scenarios as explained by the figure legend for model (13). The solid line corresponds to the analytically calculated Hopf bifurcation curve, the dotted line shows the analytically calculated loss of monotonicity curve, while the dash-dotted line acts as a visual aid to separate the weak and strong Allee effect.

the choice of the delayed underlying ecological process is an important determinant of overall population dynamics. However, a delay in negative feedback mechanisms such as intraspecific competition always results in instability and thus leads to population cycles or even to population extinction; see Figs. 2, 6, 7 and 8. We show that a delay in competition has a more dominant effect on population dynamics, compared to delays in positive feedback mechanisms arguably accounting for cooperative strategies which exert no consequences on the population's global stability. Albeit so, nontrivial transitional dynamics through self-organized formation of intermediate quasi-stationary states can be observed in models of this kind (Fig. 5). Our results are summarized in Table 1.

Note that, in the corresponding real-world population dynamics, the intermediate states predicted by model (10) may not always be seen as they would likely be blurred by the effects of stochasticity and the transient nature of the environment. These intermediate states may therefore become "hidden" in the sense that they may be difficult to observe yet they affect the population dynamics (e.g. by considerably increasing the time required to converge to the 'true' steady state). That evokes the concept of "hidden states" that has been an issue of growing importance in ecology (Clark and Bjørnstad 2004, Buckland et al. 2007). It is thought that the existence of self-organized, hidden or intermediate states can, in some cases, provide an explanation to the complexity of ecosystems' dynamics (Clark and Bjørnstad 2004). The existence of such states can sometimes be linked to the population's heterogeneity, both in terms of its age and/or physiology and in its spatial structure (e.g. So et al. 2001). In our study, however, we have shown that intermediate states can emerge in an unstructured singlespecies population as a result of the interplay between the time delayed cooperation and the 
Table 1: Summary of the results. The succession of dynamical regimes from left to right corresponds to an increase in the value of time delay $\tau$. Note that, since all models exhibit monotonous behaviour for sufficiently small $\tau$ this is not included into the table the sake of brevity.

\begin{tabular}{c|c|cccc}
\hline \hline $\begin{array}{c}\text { Model, } \\
\text { type }\end{array}$ & $\begin{array}{c}\text { Process } \\
\text { delayed }\end{array}$ & $\begin{array}{c}\text { Damped } \\
\text { oscillations }\end{array}$ & $\begin{array}{c}\text { Limit } \\
\text { cycle }\end{array}$ & $\begin{array}{c}\text { Quasistationary } \\
\text { states ('steps') }\end{array}$ & Extinction \\
\hline \hline $\begin{array}{c}\text { Eq. (5), } \\
\text { multiplicative }\end{array}$ & competition & Yes & Yes & No & No \\
\hline $\begin{array}{c}\text { Eq. (6), } \\
\text { multiplicative }\end{array}$ & cooperation & No & No & No & No \\
\hline $\begin{array}{c}\text { Eq. (10), } \\
\text { additive }\end{array}$ & cooperation & No & No & Yes & No \\
\hline $\begin{array}{c}\text { Eq. (11), } \\
\text { additive }\end{array}$ & competition & Yes & Yes & Yes \\
\hline $\begin{array}{c}\text { Eq. (12), } \\
\text { additive }\end{array}$ & competition & Yes & Yes & No & Yes \\
\hline $\begin{array}{c}\text { Eq. (13), } \\
\text { additive }\end{array}$ & $\begin{array}{c}\text { competition, } \\
\text { cooperation }\end{array}$ & Yes & Yes & Yes \\
\hline \hline
\end{tabular}

Allee effect, a novel mechanism that has not been observed before. We also mention that the original concept of hidden states is used in a purely statistical context (Buckland et al. 2007); here we show that it can arise in a deterministic model as well.

Continuous population models are sometimes considered as limiting cases of their discrete counterparts. In time discrete population models, the Allee effect is thought to have a stabilizing effect on system dynamics (in both delay and non-delay systems). In the works by Scheuring (1999) and Fowler and Ruxton (2002) logistic and Allee type difference models were presented and compared. Consequently, the per capita growth rates in Allee models were normalized to enable comparison of equivalent equilibrium stability. The inclusion of positive density dependence was shown to stabilize population dynamics, in particular with increasing Allee strength. Çelik et al. (2008) and Merdan and Gümüs (2012) confirm such findings in equivalent time delay models. In these studies, comparison was made between delayed competition models and delayed Allee effects. Analytical and numerical results suggest that even though delayed, positive density dependence remains a stabilizing mechanism. In other words, a delay in cooperation does not alter the system's stability, proving it not to be a controlling feedback as is also readily observed in the continuous case; see Fig. 4.

Biological interpretation of the 'delayed cooperation' concept is difficult to grasp, though we suggest that cooperative behaviour itself may lead to a delay in reproduction, thereby increasing the population's time to converge to it's steady state (Figs. 4 and 5). Although cooperation in nature takes many forms, one specific form are cooperative breeding populations in which 'helpers' (individuals other than parents) provide care for offspring. In addition to such alloparental care, cooperative breeding populations exhibit two further characteristics: delayed dispersal and reproductive suppression (delayed reproduction). Reproductive suppression suggests that some social or physiological factor limits the individual's ability to reproduce. Possible sources and influences can greatly vary between species, ranked individuals and sexes. Coop- 
erative breeding is a social phenomenon that has been observed in avian species (Koenig and Dickinson 2004) as well as across a rather diverse mammalian taxa (Kramer 2010): predominantly in wild canids, rodents, meerkats and some primates. Meerkats, Suricata suricatta, are highly gregarious species, obligate cooperative breeders, and live in groups of up to 50 individuals (Clutton-Brock et al. 2008). One dominant pair monolopolises reproduction, accounting for almost $80 \%$ of reproductive attempts (Griffin et al. 2003), whilst helpers of both sexes assist in rearing young. The most striking form of helping is such cooperative pup care which involves guarding pups in the natal burrow and pup feeding. Like many other cooperative breeders subordinate females commonly attempt to breed but with substantially lower reproductive success than dominant females. Behavioural tactics such as regular aggression, eviction and infanticide are employed to achieve reproductive suppression (Clutton-Brock et al. 1998, Young et al. 2006). Behavioural reproductive suppression is also seen in grey wolf packs (Asa and Valdespino 1998). Young subordinates remain in their natal pack, thus mating and reproduction are typically delayed. A common feature of cooperative breeders is that the young the individuals helped to raise become the individuals 'helpers', following their succession to breeding status in group (Wiley and Rabenold 1984). Alloparental care is particularly prominent in South American marmosets and tamarins (Solomon and French 1997). Apart from aggression as an effective mechanism limiting breeding by eviction (Snowdon and Pickhard 1999), in the common marmoset, Callithrix jacchus jacchus, physiological suppression is present, i.e. puberty is delayed in offspring that do not disperse (Abbott et al. 1981).

Cyclic dynamics in populations have stimulated a great deal of research and debate in ecology (Elton 1924, Hutchinson 1948, Krebs 1996, Krebs and Myers 1974). Contrary to the ever enlarging data sets on population cycles, population ecologists continue to debate population regulation, in particular the role of density dependence. Possibly the key issue in such disagreement is relating statistically determined patterns to ecological mechanisms responsible for population fluctuations (Williams and Liebhold 1995, Berryman and Turchin 1997). Therefore, population regulation remains a fairly contentious field, open to further investigation of the "true" governing mechanisms. A common approach used to reveal dominant underlying ecological processes is time series analysis, and there seems to be consent amongst many authors as to density dependence being one of the main causal factors of population fluctuations (Bjørnstad et al. 1998, Turchin et al. 1999, Berryman and Turchin 2001). It is well known that populations are influenced by factors that are a function of the current population density (direct density dependence) and by factors that are functions of past population densities (delayed density dependence $)^{1}$. The latter tends to destabilize population dynamics, making them more prone to cycle. There are numerous underlying causes of such second order dynamics, however the most commonly considered factors are: resource competition and cannibalism, predator-prey interactions (in particular the effect of specialist predators), parasitoids, pathogens, maternal effects, etc. Indeed, possible causes of delayed density dependence are linked to combinations of such factors, making the task of isolating one single mechanism quite difficult (Krebs 1996). A classic example of fluctuating populations are the grey-sided vole populations, Clethrionomys rufocanus, in Kilpisjarvi, Finland (Hansen et al. 1999). Attempting to reveal causes of such dynamics, Hansen et al. (1999) obtained results that show delayed density dependence

\footnotetext{
${ }^{1}$ Direct density dependence is often referred to as first order dynamics, whilst delayed density dependence is known as second (or higher) order dynamics, see Turchin (1999)
} 
is crucial for generating $3-5$ year cycles within these populations. By testing two hypotheses of community dynamics, one caused by interaction with specialist predators, and the other relating to species specific trophic interaction (overgrazing of Vaccinium) and having excluded interspecific competition, Hansen et al. (1999) show that population cycles are rather caused by intrinsic factors such as overgrazing of Vaccinium or preferential predation by least weasels on grey-sided voles. A contrasting study on field-voles (Microtus agrestis) shows that predation by weasels, is neither a sufficient nor necessary condition to initiate and drive population cycles in Kielder forest populations (Graham and Lambin 2002), suggesting more inherent mechanisms such as intraspecific competition a more possible source of fluctuations. Under the assumption cyclic behavior of both grey-sided and field vole populations is due to resource competition, we can suggest such population dynamics be described by delayed competition models such as Eq. (5). Depending on the specifics of intraspecific competition, both Eqs. (11) and (12) would be appropriate to model observed population oscillations.

Predation was also shown to be an influential force controlling population cycles in the Asian tiger mosquito, Aedes albopictus, (Walsh et al. 2012) and the southern pine beetle, Dendroctonus frontalis, (Turchin et al. 1999). A common modeling approach would consist of formulating an appropriate predator-prey model (Cushing 1976, Beretta and Kuang 1998). Nonetheless, an alternative may be found in a single species system whereby predation effects would be implicitly incorporated as additional prey mortality. Such delayed density dependent mortality was shown to oscillate for sufficiently large time delay, so Eqs. (11) and (12) would be relevant. Also, we recall here that age-dependent predator-prey interactions may cause the emergence of Allee effects, cf. De Roos and Persson 2002, De Roos et al. 2003. However, one must be very careful in making a priori generalisations based on the intuition from simple models such as ours. Furthermore, the projection of single species dynamics to two or more species systems can also be misleading. In particular, Hastings $(1983,1984)$ showed that in an age-dependent predator prey system, delays in prey recruitment are critical for the system's stability; thus, delays in higher trophic levels seem less important than indicated from single species models. Even so, delays in prey recruitment were not the sole determinant of stability, but rather the combination with the functional response. Apart from biotic factors, abiotic factors such as season length, alone, have been shown to induce delayed density dependence therefore causing population fluctuations (Smith et al. 2006).

Since the focus of this study is on single species models, our efforts are directed at intrinsic factors influencing population dynamics. Ample evidence is available on fluctuating insect populations, and suggests that cannibalism is frequently observed therein. Unlike population cycles with a period of several to many generations (small mammals, temperate forest insect pests), another type of dynamics involves fluctuations with a period approximately equal to the animal's generation or developmental time occurring usually within insect populations (seasonally synchronised life cycles). These cycles are known as generation cycles, and have been mainly studied using flour beetles (Tribolium) and phycitine moths (Pyralidae). Intensive study of flour beetles has confirmed that cannibalism is able to trigger generation cycles. Namely, in Tribolium populations, egg and pupal cannibalism by adults occurs, with pupal cannibalism occurring in lower rates (Chapman 1928, Parks 1932, Desharnais 1997). Generation cycles in Tribolium populations occur only in juvenile, not adult-life stages, that is to say that when larvae/adults are abundant they manifest egg cannibalism, so inhibiting larval-adult recruitment. Further- 
more, an increase in adult abundance leads to lower fecundity due to overcrowding effects. Both these consequences in turn result in a delayed decrease in the frequency of cannibalism, causing population fluctuations. Similar dynamics are observed in the Indian meal moth, Plodia interpunctella, in which competitive and cannibalistic effects exerted by large larvae on small larvae and/or eggs, are the main causes of delayed density dependence (Bjørnstad et al. 1998, Briggs et al. 2000). Intraspecific cannibalism seems to drive cyclic dynamics of the Indonesian lady beetle, Coccinellidae, which display clear generational cycles (Nakamura et al. 2004).

Clearly, to model cannibalistic behavior in insect populations in a comprehensive way, one should formulate a stage-structured model accounting for all developmental stages and include time delay in the appropriate mechanism (Cushing 1994). Yet delayed density dependent mortality in a simpler model such as Eqs. (11) and (12) allow for inclusion of cannibalistic effects in principle, albeit indirectly, and can be used to qualitatively describe the dynamics of one (some) of those stages, i.e. larval population dynamics. Although, we do not have equations for other life stages, Eqs. (11), (12) and (5) alone are capable of producing population cycles. Moreover, an increase in time delay in the above models leads to population oscillations, a key feature of most insect populations.

Delays may occur as a consequence of developmental time and/or interaction between individuals of different stages (as mentioned above). As most forest insects, many species of Lepidoptera exhibit cyclic population dynamics. One possible mechanism is through maternal effects, namely, the nutritional environment of the parental generation significantly influences the growth and reproductive potential of the next generation through environmentally-based maternal effects. Numerous studies on the gypsy moth, Lymantria dispar, have confirmed the existence of such effects, that is the nutritional experience of the parental generation affects the length of the pre-feeding period, development time and pupal weight in the subsequent generation (Rossiter 1991). These traits are thought to be critically important in insect population dynamics because of their influence on natality and mortality. Moreover, these effects take place with a time lag, and are able to destabilize the population, leading to the occurrence of cyclic behavior (Ginzburg and Taneyhill 1994). Ginzburg and Taneyhill (1994) developed a time discrete maternal effect system coupled with delayed logistic growth and fitted the results to data of six species of forest moths (including gypsy moths). The maternal model predicts cycle periods very close to observed values; however, an important factor that has been shown to influence gypsy moth populations has been overlooked - namely, the the Allee effect (Liebhold and Bascompte 2003 Tobin et al. 2009, Vercken et al. 2011). Thus, an interesting future extension to this work would be to test multi-annual gypsy moth dynamics with a time continuous maternal model including a delayed Allee effect such as given by Eqs. (5), (11), (12) and (13).

We emphasize that the interplay between time delay and the Allee effect can be subtle and rather counterintuitive. In our study, we revisit the notable destabilizing effect of time delays, as for a fixed Allee threshold an increase in time delay leads to oscillatory behavior, in most models. However, depending on the particular description of the delayed process, the shape of the bifurcation curves can change significantly; see Figs. 2, 6 and 7. In all three corresponding models the same underlying ecological process (i.e. competition) is subject to delay, yet the results are considerably different. In addition, the possibility of the population driven to extinction occurs only in the additive formulation of the Allee effect (Figs. 6, 7 and 8) but not in the multiplicative one (Fig. 2). Also, the pattern of population growth can be remarkably different depending on 
whether the time delayed cooperation is described by multiplicative or additive formulations of the model, cf. Figs. 4 and 5. These results indicate strong dependence on the choice of functional form (parametrization), thereby evoking the controversial issue of model sensitivity (Adamson and Morozov 2012, Fussmann and Blasius 2005).

The majority of population fluctuations are described by the generally accepted specialist predator (or parasitoid) hypothesis, with just a few exceptional cases involving other kinds of trophic interactions (Turchin 2003). This of course does not guarantee nor imply that intrinsic controlling mechanisms are completely absent in populations. However, to date such inherent hypotheses have not fully provided theoretically sound and empirically supported population models (due to tenuous connections to data). We emphasise that our intention was not to develop a comprehensive framework or provide a unique solution to population cycles, but rather to demonstrate and gain informative insight into the consequences of delayed feedback mechanisms and their interaction with density dependence through the Allee effect. Time delays are integral parts of ecological systems, and as such may aid in explaining underlying mechanisms of complex population dynamics such as population fluctuations. A common standpoint is that in stable (non-oscillating) populations these time lags are insufficiently large to cause fluctuations, therefore are disregarded. We hereby tentatively propose that even non-fluctuating populations may be subject to delayed processes that do not alter population stability, such as positive density dependent mechanisms, i.e. cooperation.

Acknowledgements. S.P. gratefully acknowledges the support given by the University of Leicester in granting an academic study leave which was essential for completion of this work. 


\section{A Linear stability analysis}

The general approach adopted in this and the following appendix is based on the standard linearization technique coupled with basic knowledge of ODE theory used to obtain a bifurcation value of the time delay parameter, $\tau$. Since the method in question can be easily extended and applied to all models introduced in this paper, stability analysis will be shown for one model as an illustrative example, and results for other models listed. Stability of the upper positive equilibrium is considered as it is known that the intermediate equilibrium $U_{*}=\beta$ is always unstable.

Suppose $x$ is a small perturbation from the steady state $U_{*}=K=1$ :

$$
x=U-U_{*}
$$

To determine equilibrium stability we investigate the behaviour of this small perturbation, whether it grows or decays, and for that purpose we linearize around the steady state, ignore higher order terms of model (5), which gives:

$$
\frac{d x}{d t}=(\beta \gamma-\gamma) x_{\tau}
$$

Note that we treat the delayed variables, $U(t-\tau)=U_{\tau}$ and $x(t-\tau)=x_{\tau}$ as separate variables throughout linearising. We look for solutions of form $x=c e^{\lambda t}$, where $c$ is a constant and $\lambda$ are the eigenvalues of the system which determine its stability. By substituting this solution form in (15) we obtain the transcendental characteristic equation:

$$
\lambda=(\beta \gamma-\gamma) e^{-\lambda \tau},
$$

for which analytical solutions are difficult to find. Nevertheless, from a stability viewpoint it is important to find whether there are any solutions with $\operatorname{Re} \lambda>0$ which implies instability (since the perturbation grows exponentially with time). By setting $\lambda=i \omega$, we assume $\operatorname{Re} \lambda=0$, and obtain an expression for the critical value at which the system passes through the Hopf bifurcation, below which stability prevails and above which instability occurs. Substituting into (16) and separating real and imaginary parts of the transcendental equation, we obtain the system:

$$
\begin{aligned}
& -(\beta \gamma-\gamma) \cos (\omega \tau)=0 \\
& \omega=-(\beta \gamma-\gamma) \sin (\omega \tau)
\end{aligned}
$$

Following some elementary calculations we are able to find the critical value of $\tau$ :

$$
\tau_{c}=-\frac{\pi}{2(\beta \gamma-\gamma)}
$$

In all subsequent analysis, the characteristic growth rate was taken as $\gamma=1$. In the below table we summarize stability conditions for all other models used. 


\begin{tabular}{|c|c|}
\hline Model & Stability Condition \\
\hline$\gamma U\left(U_{\tau}-\beta\right)(K-U)$ & $\lambda=\beta-1$ \\
$\gamma U\left(-\beta+(1+\beta) U_{\tau}-U^{2}\right)$ & $\tau_{c}=\frac{\arccos \left(\frac{2}{1+\beta}\right)}{\sqrt{\frac{1+\beta}{2}}}$ \\
$\gamma U\left(-\beta+(1+\beta) U-U_{\tau} U\right)$ & $\tau_{c}=\frac{\arccos \beta}{\sin (\arccos \beta)}$ \\
$\gamma U\left(-\beta+(1+\beta) U-U_{\tau}^{2}\right)$ & $\tau_{c}=\frac{\arccos \left(\frac{\beta+1}{2}\right)}{\sqrt{4-(\beta+1)^{2}}}$ \\
$\gamma U\left(-\beta+(1+\beta) U_{\tau}-U_{\tau}^{2}\right)$ & $\tau_{c}=-\frac{\pi}{2(\beta-1)}$ \\
\hline
\end{tabular}

Table 2: Note that $U(t)=U, U(t-\tau)=U_{\tau}$ and parameter $\gamma=1$.

For the delayed cooperation model (6) stability analysis confirmed that the equilibrium remains stable as:

$$
\lambda=\beta-1
$$

thus the eigenvalue is always negative for all biologically viable values of $\beta$. Loss of stability does not occur for the model incorporating time delay in the maturation term (10), as the stability condition in this case reads:

$$
\lambda=\frac{\arccos \left(\frac{2}{1+\beta}\right)}{\sqrt{\frac{1+\beta}{2}}}
$$

Since $-1 \leq \beta \leq 0.5, \arccos \left(\frac{2}{1+\beta}\right)$ is not defined, there are no bifurcation values, $\tau_{c}$. As for the model incorporating time delay in direct competition (11) we were able to map all critical values, $\tau_{c}$ :

$$
\tau_{c}=\frac{\arccos \beta}{\sin (\arccos \beta)}
$$

with the exception of $\beta=-1$ as it is a singularity. Addition of another delayed term into direct competition results in model (12) for which the stability condition reads:

$$
\tau_{c}=\frac{\arccos \left(\frac{\beta+1}{2}\right)}{\sqrt{4-(\beta+1)^{2}}}
$$

Analytically defining bifurcation values, $\tau_{c}$, for the model with two delayed terms (13) the same as in model with delayed competition (5).

\section{B Loss of monotonicity analysis}

Following the linearization method previously described, we obtain expressions (and subsequently values) for the time delay parameter, $\tau$, when the system loses monotonicity, i.e. when the solution still approaches the stable equilibrium $U_{*}=K$, but in a nonmonotonous manner (referred to as damped oscillations). We introduce the conditions of monotonicity, solving for one model, and list results for all others, as above.

We consider model (5) and its corresponding characteristic equation:

$$
F(\lambda ; \tau)=\lambda+e^{-\lambda \tau}(\gamma-\beta \gamma)=0
$$




\begin{tabular}{|c|c|}
\hline Model & Stability Condition \\
\hline$\gamma U\left(U_{\tau}-\beta\right)(K-U)$ & $\lambda=\beta-1$ \\
$\gamma U\left(-\beta+(1+\beta) U_{\tau}-U^{2}\right)$ & $\frac{1}{\tau_{*}(\beta+1)}+e^{1+2 \tau_{*}}=0$ \\
$\gamma U\left(-\beta+(1+\beta) U-U_{\tau} U\right)$ & $\frac{1}{\tau_{*}}-e^{1-\beta \tau_{*}}=0$ \\
$\gamma U\left(-\beta+(1+\beta) U-U_{\tau}^{2}\right)$ & $\frac{1}{2 \tau_{*}}-e^{1-(1+\beta) \tau_{*}}$ \\
$\gamma U\left(-\beta+(1+\beta) U_{\tau}-U_{\tau}^{2}\right)$ & $\tau_{*}=\frac{1}{e(1-\beta)}$ \\
\hline
\end{tabular}

Table 3: Note that $U(t)=U, U(t-\tau)=U_{\tau}$ and parameter $\gamma=1$.

In general, whether the solution is monotone or not depends on the roots of this eigenvalue equation and loss of monotonicity is associated with the total loss of all relevant real eigenvalues. Obviously, when $\tau=0$, the eigenvalue is negative for all biologically relevant values of $\beta$, with the exception for $\beta=-1$, in which case stability of system cannot be inferred. By increasing the time delay, the eigenvalue goes complex, at a value we will denote by $\tau_{*}$. At this critical value the two curves intersect and the following condition holds:

$$
\frac{\partial F}{\partial \lambda}(\lambda ; \tau)=0
$$

On elimination of $\lambda$, using elementary calculus, one finds an explicit condition for $\tau$ :

$$
\tau_{*}=\frac{1}{e(\gamma-\beta \gamma)}
$$

and so loss of monotonicity is predicted for $\tau \geq \tau_{*}$. Note this is the only case in which we may obtain an explicit expression, as with all other models implicit expressions are presented. In the below table we summarize all critical values, $\tau_{*}$ for which solutions lose monotonicity, for all subsequent models.

In Eq. (6) the solution remains monotonous as the eigenvalues are always negative for all applicable values of the Allee threshold $(\lambda=\beta-1)$.

Model (10), yielding the most interesting step-like results, clearly preserves monotonicity throughout all possible values of the time delay parameter, $\tau$, as the monotonicity condition does not have any roots, and reads:

$$
\frac{1}{\tau(\beta+1)}+e^{1+2 \tau}=0
$$

For model (11) the implicit expression for the critical time delay is:

$$
\frac{1}{\tau}-e^{1-\beta \tau}=0
$$

A differing monotonicity condition is obtained for a fully delayed density dependent mortality (model (12)):

$$
\frac{1}{2 \tau}-e^{1-(1+\beta) \tau}
$$

Our two delay model yielded the same condition as in model (5), as was expected and confirmed in numerical simulations. 


\section{References}

[1] Abbott O H, McNeilly A S, Lunn S F, Hulme M J, Burden F J (1981) Inhibition of ovarian function in subordinate marmoset monkeys (Callithrix jacchus jacchus). Journal of Reproduction and Fertility 63(2):335-345.

[2] Adamson M W, Morozov A Y (2013) When can we trust our model predictions? Unearthing structural sensitivity in biological systems. Proceedings of the Royal Society A: Mathematical, Physical and Engineering Sciences 469 (2149).

[3] Amarasekara P (1998a) Interactions between local dynamics and dispersal: Insights from single species models. Theoretical Population Biology 53(1):44-59.

[4] Amarasekara P (1998b) Allee effects in metapopulation dynamics. The American Naturalist $152(2): 298-302$.

[5] Asa C S, Valdespino C (1998) Canid reproductive biology: an integration of proximate mechanisms and ultimate causes. American Zoologist 38(1):251-259.

[6] Beddington J R, May R M (1975) Time delays are not necessarily destabilizing. Mathematical Biosciences 27:109-117.

[7] Beretta E, Kuang Y (1998) Global analyses in some delayed ratio-dependent predator-prey systems. Nonlinear Analysis: Theory, Methods and Applications 32(3): 381-408.

[8] Berryman A, Turchin P (2001) Identifying the density-dependent structure underlying ecological time series. Oikos 92:265-270.

[9] Berryman A, Turchin P (1997) Detection of delayed density dependence: comment. Ecology 78:318-320.

[10] Bjørnstad O N, Begon M, Stenseth N C, Falk W, Sait S M, Thompson D J (1998) Population dynamics of the Indian meal moth: Demographic stochasticity and delayed regulatory mechanisms. Journal of animal ecology 67: 110-126.

[11] Boukal D S, Berec L (2002) Single species models of the Allee effect: Extinction boundaries, sex ratios and mate encounters. Journal of Theoretical Biology 218:375-394.

[12] Briggs C J, Sait S M, Begon M, Thompson D J, Godfray H C J (2000) What causes generation cycles in populations of stored-product moths? Journal of Animal Ecology 69(2):352-366.

[13] Cao Y, Gard T C (1995) Ultimate bounds and global asymptotic stability for differential delay equations. Rocky Mountain Journal of Mathematics 25(1):119-131.

[14] Çelik C, Merdan H, Duman O, Akin Ö (2008) Allee effects on population dynamics with delay. Chaos, Solitons and Fractals 37:65-74.

[15] Chapman R N (1928) The quantitative analysis of environmental factors. Ecology 9:111-122. 
[16] Clutton-Brock T H, Brotherton P N M, Smith R, McIlrath G M, Kansky R, Gaynor D, O'Riain M J, Skinner J D (1998) Infanticide and expulsion of females in a cooperative mammal. Proceedings: Biological Sciences 265(1412):2291-2295.

[17] Clutton-Brock T H, Hodge S J, Flower T P (2008) Group size and the suppression of subordinate reproduction in Kalahari meerkats. Animal Behaviour 76(3):689-700.

[18] Cooke K L, Grossman Z (1982) Discrete delay, distributed delay and stability switches. Journal of Mathematical Analysis and Applications 86:592-627.

[19] Courchamp F, Berec L, Gascoigne J (2008) Allee effects in Ecology and Conservation. Oxford University Press.

[20] Courchamp F, Clutton-Brock T, Grenfell B (1999) Inverse density dependence and the Allee effect. Trends in Ecology and Evolution 14(10):405-410.

[21] Cushing J M (1976) Predator-prey interactions with time delays. Journal of Mathematical Biology (3-4):369-380.

[22] Cushing J M (1994) Oscillations in age-structured population models with an Allee effect. Journal of Computational and Applied Mathematics 52:71-80.

[23] De Roos A M, Persson L (2002) Size-dependent life-history traits promote catastrophic collapses of top predators. Proceedings of the National Academy of Sciences 99(20):1290712912 .

[24] De Roos A M, Persson L, Thieme H R (2003) Emergent Allee effects in top predators feeding on structured prey populations. Proceedings of the Royal Society B 270:611-618.

[25] Dennis B (1989) Allee effects: Population growth, critical density and the chance of extinction. Natural Resource Modeling 3:481-538.

[26] Desharnais R A (1997) Population dynamics of Tribolium. Pages 303-328 in Structured Population Models in Marine, Terrestrial, and Freshwater Systems, S. Tuljapurkar and H. Caswell (editors), Chapman \& Hall, New York.

[27] Elton C S (1924) Periodic fluctuations in the number of animals: their causes and effects. British Journal of Experimental Biology 2:119-163.

[28] Fagan W F, Lewis M A, Neurbert M G, van der Driessche P (2002) Invasion theory and biological control. Ecol. Lett. 5:148157.

[29] Fowler M S, Ruxton G D (2002) Population dynamic consequences of Allee effects. Journal of Theoretical Biology 215:39-46.

[30] Freedman H I, Gopalsamy K (1986) Global stability in time delayed single species dynamics. Bulletin of Mathematical Biology 48(5):485-492.

[31] Fussmann GF, Blasius B (2005) Community response to enrichment is highly sensitie to model structure. Biology Letters 1:9-12. 
[32] Ginzburg L R, Taneyhill D E (1994) Population cycles of forest Lepidoptera: A maternal effect hypothesis. Journal of Animal Ecology 63:79-92.

[33] Gonzales-Andujar J L, Fernandes Quintanilla C, Navarrete L (2006) Population cycles produced by delayed density dependence in an annual plant. The American Naturalist 168:318-322.

[34] Gopalsamy K, Ladas G (1990) On the oscillation and asymptotic behaviour of $\dot{N}(t)=$ $N(t)\left[a+b N(t-T)-c N^{2}(t-T)\right]$. Quarterly of Applied Mathematics 3:433-440.

[35] Graham I S, Lambin X (2002)The impact of weasel predation on cyclic field vole survival: The specialist predator hypothesis contradicted. Journal of Animal Ecology 71(6):946-956.

[36] Griffin A S, Pemberton J M, Brotherton P N M, McIlrath G, Gaynor D, Kansky R, O'Riain J, Clutton-Brock T H (2003) A genetic analysis of breeding success in the cooperative meerkat (Suricata suricatta). Behavioural Ecology 14:472-480.

[37] Griffiths S W, Armstrong J D, Metcalfe N B (2003) The cost of aggregation: Juvenile salmon avoid sharing winter refuges with siblings. Behavioural Ecology 14(5):202-206.

[38] Hansen T F, Stenseth N C, Henttonen H, Tast J (1998) Interspecific and intraspecific competition as causes of direct and delayed density dependence in a fluctuating vole population. Proceedings of the National Academy of Sciences of the United States of America 96(3):986-991.

[39] Hutchinson G E (1948) Circular causal systems in ecology. Annals of the New York Academy of Sciences 50:221-246.

[40] Koenig W D, Dickinson J L (2004) Ecology and evolution of cooperative breeding in birds. Cambridge University Press.

[41] Kramer A M, Dennis B, Liebhold A M, Drake J M (2009) The evidence for Allee effects. Population Ecology 51:341-354.

[42] Kramer K L (2010) Cooperative breeding and its significance to the demographic success of humans. Annual Review of Anthropology 39:417-436.

[43] Krebs C J (1996) Population cycles revisited. Journal of Mammalogy 77(1):8-24.

[44] Krebs C J and Myers J (1974) Population cycles in small mammals. Advances in Ecological Research 8:267-399.

[45] Kuang Y (1993) Delay differential equations with applications in population dynamics. Academic Press, New York.

[46] Lewis M A, Kareiva P (1993) Allee dynamics and the spread of invading organisms. Theoretical Population Biology 43:141-158.

[47] Lewis M A, van den Driessche P. (1993). Waves of extinction from sterile insect release. Mathematical Bioscience 116:221-247. 
[48] Levins R (1969) Some demographic and genetic consequences of environmental heterogeneity for biological control. Bull. Entomol. Soc. Am. 15:237240.

[49] Liebhold A M, Bascompte J (2003) The Allee effect, stochastic dynamics and the eradication of alien species. Ecology Letters 6:133-140.

[50] Liebhold A M, Tobin P C (2010) Exploiting the Achilles heels of pest invasions: Allee effects, stratified dispersal and management of forest insect establishment and spread. New Zealand Journal of Forestry Science 40:S25-S33.

[51] Liz E, Pinto M, Robledo G, Trofimchuk S, Tkachenko V (2003) Wright type delay differential equations with negative Schwarzian. Discrete and Continuous Dynamical Systems 9:309321.

[52] May R M (1973) Time delay versus stability in population models with two and three trophic levels. Ecology 54(2):315-325.

[53] Maynard Smith J (1974) Models in Ecology. Cambridge University Press.

[54] Merdan H, Duman O, Akin Ö, Çelik C (2009) Allee effects on population dynamics in continuous (overlapping) case. Chaos, Solitons and Fractals 39:1994-2001.

[55] Merdan H, Gümüş Ö (2012) Stability analysis of a general discrete-time population model involving delay and Allee effects. Applied Mathematics and Computation 219:1821-1832.

[56] Morozov A Y, Petrovskii S V, Li B-L (2006) Spatiotemporal complexity of the patchy invasion in a predatorprey system with the Allee effect. J. Theor. Biol. 238:1835.

[57] Nakamura K, Hasan N, Abbas I H, Godfray C J, Bonsall M B (2004) Generation cycles in Indonesian lady beetle populations may occur as a result of cannibalism. Proceedings: Biological Sciences 271(6):S501-S504

[58] Nakaoka S, Wang W, Takeuchi Y (2009) Effect of parental care and aggregation on population dynamics. Journal of Theoretical Biology 260:161-171.

[59] Park T (1932) Studies in population physiology: the relation of numbers to initial population growth in the flour beetle Tribolium confusum Duval. Ecology 13:172-181.

[60] Petrovskii S V, Morozov A Y, Venturino E (2002) Allee effect makes possible patchy invasion in a predator-prey system. Ecology Letters 5:345-352.

[61] Petrovskii S V, Malchow H, Hilker F M, Venturino E (2005a) Patterns of patchy spread in deterministic and stochastic models of biological invasion and biological control. Biological Invasions 7:771-793.

[62] Petrovskii S V, Morozov A Y, Li B L (2005b) Regimes of biological invasion in a prey predator system with the Allee effect. Bulletin of Mathematical Biology 67:637-661.

[63] Petrovskii S, Blackshaw R, Li B L (2008) Consequences of the Allee effect and intraspecific competition on population persistence under adverse environmental conditions. Bulletin of Mathematical Biology 70 (2):412-437. 
[64] Rossiter M C (1991) Environmentally-based maternal effects: A hidden force in insect population dynamics. Oecologia 87(2):288-294.

[65] Ruan S G (1995) The effect of delays on stability and persistence in plankton models. Nonlinear Analysis - Theory, Methods \& Applications 24, 575-585.

[66] Scheuring I (1999) Allee effect increases the dynamical stability of populations. Journal of Theoretical Biology 199:407-414.

[67] Smith M J, White A, Lambin X, Sheratt J A, Begon M (2006) Delayed-density dependent season length alone can lead to rodent population cycles. The American Naturalist 167(5): 695-704.

[68] Snowdon C T, Pickhard J J (1999) Family feuds: Severe aggression among cooperatively breeding cotton top tamarins. International Journal of Primatology 20(5):651-663.

[69] So J W H, Wu J, Zou W (2001) Structured population on two patches: modeling dispersal and delay. Journal of Mathematical Biology 43:37-51.

[70] Solomon N G, French J A (1997) Cooperative breeding in mammals. Cambridge University Press.

[71] Stephens P A, Sutherland W J, Freckleton R P (1999) What is the Allee effect? Oikos 87(1):185-190.

[72] Taylor C M, Hastings A (2005) Allee effects in biological invasions. Ecology letters 8:895908.

[73] Terry A J (2011) Dynamics of a structured population on two patches. Journal of Mathematical Analysis and Applications 378:1-15.

[74] Tobin P C, Robinet C, Johnson D M, Whitmire S L, Bjørnstad O N, Liebhold A M (2009) The role of Allee effects in gypsy moth(Lymantria dispar (L.)) invasions. Population Ecology 51:373-384.

[75] Turchin P (1999) Population regulation: A synthetic view. Oikos 84(1):153-159.

[76] Turchin P (2003) Complex population dynamics: A theoretical/empirical synthesis. Princeton University Press.

[77] Turchin P, Taylor A D, Reeve J D (1999) Dynamical role of predators in population cycles of a forest insect: an experimental test. Science 285 (5430):1068-1071.

[78] Van Kooten T, De Roos A M, Persson L (2005) Bistability and an Allee effect as emergent consequences of stage-specific predation. Journal of Theoretical Biology 237:67-74.

[79] Vercken E, Kramer A M, Tobin P C, Drake J M (2011) Critical patch size generated by Allee effect in gypsy moth, Lymantria dispar (L.). Ecology Letters 14:179-186.

[80] Walsh R K, Bradley C, Apperson C S, Gould F (2012) An experimental field study of delayed density dependence in natural populations of Aedes albopictus. PLoS ONE 7(4): e35959. doi:10.1371/journal.pone.0035959. 
[81] Wiley H R, Rabenold K N (1984) The evolution of cooperative breeding by delayed reciprocity and queuing for favourable social positions. Evolution 38(3):609-621.

[82] Williams D W, Liebhold A M (1995) Detection of delayed density dependence: effects of autocorrelation in an exogenous factor. Ecology 76:1005-1008.

[83] Young A J, Carlson A A, Monfort S L, Russell A F, Bennett N C, Clutton-Brock T H (2006) Stress and the suppression of subordinate reproduction in cooperatively breeding meerkats. Proceedings of the National Academy of Sciences of the United States of America 103(32):12005-12010. 